\title{
Doubly charged Higgsinos at the Tevatron
}

\author{
Durmuş A. Demir, ${ }^{1, *}$ Mariana Frank, ${ }^{2, \dagger}$ Dilip Kumar Ghosh, ${ }^{3, \ddagger}$ Katri Huitu, ${ }^{4, \S}$ Santosh Kumar Rai, ${ }^{4, \|}$ and Ismail Turan ${ }^{2, \pi}$ \\ ${ }^{1}$ Department of Physics, Izmir Institute of Technology, IZTECH, TR35430 Izmir, Turkey \\ ${ }^{2}$ Department of Physics, Concordia University, 7141 Sherbrooke Street West, Montreal, Quebec, Canada H4B 1 R6 \\ ${ }^{3}$ Department of Theoretical Physics and Centre for Theoretical Sciences, Indian Association for the Cultivation of Science, \\ $2 A \& 2 B$ Raja S.C. Mullick Road, Kolkata 700 032, India \\ ${ }^{4}$ Department of Physics, University of Helsinki, and Helsinki Institute of Physics, \\ Post Office Box 64, FIN-00014 University of Helsinki, Finland
}

(Received 27 March 2009; published 11 May 2009)

\begin{abstract}
Several supersymmetric models with extended gauge structures, motivated by either grand unification or by neutrino mass generation, predict light doubly charged Higgsinos. In this work we study the signals of doubly charged Higgsinos at the Tevatron in both pair- and single-production modes, and show that it is possible, especially from the events containing same-sign same-flavor isolated leptons, to disentangle the effects of doubly charged Higgsinos in the Tevatron data.
\end{abstract}

DOI: 10.1103/PhysRevD.79.095006

PACS numbers: 12.60.Jv, 12.60.Fr

\section{INTRODUCTION}

The long-awaited Large Hadron Collider (LHC), while delayed by technical problems, is expected to start soon. At the beginning, it is very likely that the LHC will run at a low beam energy $(\approx 10 \mathrm{TeV})$ and low luminosity $(\approx$ $10^{32} \mathrm{~cm}^{-2} \mathrm{~s}^{-1}$ ) regime. The data collected during this period will be mainly used to calibrate different components of the detectors involved in this complicated experiment. In addition to the detector calibration, the early measurements of $W$ and $Z$ production cross sections will be able to provide a precise knowledge of different parton densities at such high energies. While the LHC may take at least one or two years to provide us the first glimpse of the Higgs boson of the standard model (SM) or any signature of new physics beyond the SM, it is worth exploring the physics potential of the currently running largest energy collider facility, Tevatron at Fermilab. It should be noted that the Tevatron experiments D0 and CDF have each recorded over $4 \mathrm{fb}^{-1}$ (Fall 2008) data [1]. Very recently, the combined analysis from D0 and CDF experiments based on data samples with luminosities between $1.7-3 \mathrm{fb}^{-1}$ excluded a SM Higgs boson with a mass of $170 \mathrm{GeV}$ at $95 \%$ C.L. [1]. It has been speculated that the Tevatron will run through 2010 and at the current pace, it is aiming for over $8 \mathrm{fb}^{-1}$ of data collection by these two experiments by the end of 2010 [1]. With these huge data sets, the Tevatron will be able to probe the SM Higgs boson masses from 145 to $185 \mathrm{GeV}$. In addition to the Higgs search, one can take advantage of this data set to explore different scenarios of physics beyond the $\mathrm{SM}$ at the $\mathrm{TeV}$

\footnotetext{
*demir@physics.iztech.edu.tr

mfrank@alcor.concordia.ca

‡dilipghoshjal@gmail.com

\$huitu@cc.helsinki.fi

"santosh.rai@helsinki.fi

Iituran@physics.concordia.ca
}

scale. The supersymmetric left-right model (which we hereon call "LRSUSY") is one such example, which is based on the gauge group $S U(3)_{C} \times S U(2)_{L} \times S U(2)_{R} \times$ $U(1)_{B-L}$. The LRSUSY model naturally arises from highscale models unifying left- and right-handed matter, such as superstrings [2] or supersymmetric grand unified theories like $E_{6}$ or $S O(10)$ [3]. The LRSUSY model, where the $S U(2)_{R}$ gauge symmetry is broken by a triplet Higgs field with quantum numbers $B-L= \pm 2$, has several attractive features:

(1) In LRSUSY, $R$ parity $\left[R=(-1)^{(3 B+L+2 S)}\right]$ is automatically conserved (with $B, L$, and $S$ baryon, lepton, and spin quantum numbers, respectively), since the $B-L$ is part of the gauge symmetry [4]. Therefore, the lightest supersymmetric particle (LSP) is automatically stable and it qualifies as a candidate for cold dark matter [5]. Therefore events with supersymmetric particles are generically accompanied by missing energy [6], a component taken away by the LSP.

(2) In LRSUSY, strong and weak $C P$ problems, the two serious naturalness problems in which the minimal supersymmetric model (MSSM) suffers, are naturally avoided [7].

(3) In LRSUSY, when $S U(2)_{R}$ is broken by a triplet Higgs, the neutrino masses can be generated in a natural way by allowing for $R$-parity violating couplings through spontaneous $R$-parity violation, or by invoking the seesaw mechanism [8]. The seesaw mechanism is natural in this model, where a Higgs triplet provides the Majorana mass term for the neutrinos (a detailed account of these aspects can be found in [7,9-11]).

The Higgs triplets consist of doubly charged, singly charged, and neutral fields. By supersymmetry, each superfield has bosonic (Higgs bosons) and fermionic (Higgsinos) degrees of freedom. The doubly charged fields 
cannot mix with fields belonging to other electric charge sectors. The doubly charged Higgsinos, as for any fermionic component in a supermultiplet, cannot obtain soft SUSY-breaking masses, and their masses thus originate solely from the superpotential. Indeed, the superpotential involves bilinears of the Higgs triplets, and the corresponding $\mu$ parameter (in the language of MSSM) generates the requisite masses for doubly charged Higgsinos. In general, there is no telling whether the $\mu$ parameter lies at the electroweak scale (similar to the $\mu$ problem of the MSSM); however, if it does, then Higgsinos weigh within the reach of present colliders [12]. The $\mu$ parameter can be generated and stabilized at the weak scale though some variation of the Giudice-Masiero mechanism [13] or some dynamical stabilization mechanism utilizing an extra gauge group, under which the charges of left and right triplets do not sum up to zero [14].

The collider signals of the doubly charged Higgs bosons of the LRSUSY model have been studied in the context of upcoming collider experiments [15]. The signatures of the fermionic partners of the doubly charged Higgs bosons have been studied at the LHC [16,17] and at a linear collider [11,18]. Moreover, signatures of such doubly charged Higgsinos of the LRSUSY model have been explored previously [19], and in the framework of a gauge mediated supersymmetry breaking scenario, at the Tevatron. In this paper, we consider the pair and single production of doubly charged Higgsinos at the Tevatron and their subsequent decays which lead to multilepton plus large missing energy signatures. We perform a background analysis and show how the signal can be extracted. We then discuss the discovery potential of the Tevatron and demonstrate that it can probe doubly charged Higgsino masses up to $200-300 \mathrm{GeV}$ at the present integrated luminosity.

The rest of the paper is organized as follows. In Sec. II we describe the salient features of the LRSUSY model. In Sec. III we discuss the pair and single production of doubly charged Higgsinos at the Tevatron energies and their decay channels. We analyze thoroughly the signal and background events and discuss the possible discovery limits for doubly charged Higgsinos using the multilepton signature. Finally, our conclusions are given in Sec. IV.

\section{DOUBLY CHARGED HIGGSINOS IN THE LEFT- RIGHT SUPERSYMMETRIC MODEL}

In what follows we choose LRSUSY to describe the interactions of the doubly charged Higgsinos, although we expect our analysis to be general enough to hold in generic models (such as the 3-3-1 model) which predict such exotic particles. LRSUSY adds supersymmetry to the left-right gauge symmetry group

$$
G_{L R} \equiv S U(2)_{L} \times S U(2)_{R} \times U(1)_{B-L}
$$

in addition to color $S U(3)$. The matter spectrum consists of three generations of quark and lepton superfields as well as the gauge bosons of each group factor [10]. The Higgs sector is spanned by

(i) Two $B-L=0$ Higgs bidoublets: $\Phi_{1}(2,2,0)$ and $\Phi_{2}(2,2,0)$ required to generate nonvanishing Cabibbo-Kobayashi-Maskawa quark mixing.

(ii) Two $B-L=-2$ Higgs triplets: $\Delta_{L}(3,1,-2)$ and $\Delta_{R}(1,3,-2)$. One is required to break $S U(2)_{R} \times$ $U(1)_{B-L}$ down to $U(1)_{Y}$ spontaneously and both are needed to preserve left-right symmetry.

(iii) Two $B-L=+2$ Higgs triplets: $\delta_{L}(3,1,2)$ and $\delta_{R}(1,3,2)$ required to cancel the anomalies.

Given this superfield spectrum, the superpotential of LRSUSY takes the form

$$
\begin{aligned}
W= & \mathbf{Y}_{Q}^{(i)} Q^{T} \tau_{2} \Phi_{i} \tau_{2} Q^{c}+\mathbf{Y}_{L}^{(i)} L^{T} \tau_{2} \Phi_{i} \tau_{2} L^{c} \\
& +i \mathbf{Y}_{L L}\left[L^{T} \tau_{2} \delta_{L} L+L^{c T} \tau_{2} \Delta_{R} L^{c}\right] \\
& +\mu_{3} \operatorname{Tr}\left[\Delta_{L} \delta_{L}+\Delta_{R} \delta_{R}\right]+\mu^{(i j)} \operatorname{Tr}\left[\tau_{2} \Phi_{i}^{T} \tau_{2} \Phi_{j}\right] \\
& +W_{\mathrm{NR}}
\end{aligned}
$$

where generation indices are suppressed. $W_{\mathrm{NR}}$ stands for possible nonrenormalizable operators. The dimensionful parameters $\mu$ and $\mu^{(i j)}$ will be taken to have been stabilized at the electroweak scale without specifying the responsible mechanism [13,14]. The $\mathbf{Y}_{Q}^{(i)}$ and $\mathbf{Y}_{L}^{(i)}$ are, respectively, the Yukawa matrices for quarks and leptons for their couplings to the bidoublet $\Phi_{i}$. The $\mathbf{Y}_{L L}$ parametrizes couplings of left- and right-handed leptons to the corresponding Higgs triplet. The left-right symmetry enforces $\mathbf{Y}_{Q, L}$ matrices to be Hermitian and the $\mathbf{Y}_{L L}$ matrix to be symmetric in the space of fermion generations.

The parity and $S U(2)_{R}$ symmetries are spontaneously broken by the nonvanishing vacuum expectation values (VEVs) of the Higgs mutiplets

$$
\begin{gathered}
\left\langle\Delta_{L, R}\right\rangle=\left(\begin{array}{cc}
0 & v_{\Delta_{L, R}} \\
0 & 0
\end{array}\right), \quad\left\langle\delta_{L, R}\right\rangle=\left(\begin{array}{cc}
0 & 0 \\
v_{\delta_{L, R}} & 0
\end{array}\right), \\
\left\langle\Phi_{1,2}\right\rangle=\left(\begin{array}{cc}
v_{1,2} & 0 \\
0 & v_{1,2}^{\prime}
\end{array}\right)
\end{gathered}
$$

where the position of various nonvanishing entries is dictated by the electric charge conservation. Clearly, the VEVs of the bidoublets $v_{1,2}$ and $v_{1,2}^{\prime}$ are responsible for giving mass to quarks and leptons. The VEV $v_{\delta_{L}}$ must be exceedingly small for neutrino masses to be generated correctly (if $\mathbf{Y}_{L L}$ is not taken unnaturally small) and for the $\rho$ parameter to stay close to unity (which also constrains $v_{\Delta_{L}}$ to be small). The VEV $v_{\Delta_{R}}$ must lie close to the right-handed neutrino scale if neutrino masses are to be generated by the seesaw mechanism. If $v_{\Delta_{R}}$ along with $v_{\delta_{R}}$ is chosen to stay close to the TeV domain if $W_{R}$ and $Z_{R}$ are to be seen at collider experiments, one must seek alternative ways to generate neutrino masses.

Though $\Delta_{L}$ is not necessary for symmetry breaking [15] as it is introduced only for preserving left-right symmetry, 
both $\Delta_{L}^{--}$and its right-handed counterpart $\Delta_{R}^{--}$play very important roles in phenomenological studies of the LRSUSY model. It has been shown that these bosons, and their fermionic counterparts, could be sufficiently light [12] to be reachable in present colliders. As our analysis deals primarily with the doubly charged Higgsinos, we hereon focus on these doubly charged states.

The mass terms of doubly charged Higgsinos read as

$$
\mathcal{L}_{\Delta \delta}^{\operatorname{mass}}=-M_{\tilde{\Delta}_{L}^{--}} \tilde{\Delta}_{L}^{--} \tilde{\delta}_{L}^{++}-M_{\tilde{\Delta}_{R}^{--}} \tilde{\Delta}_{R}^{--} \tilde{\delta}_{R}^{++}
$$

where

$$
M_{\tilde{\Delta}_{L}^{--}}=M_{\tilde{\Delta}_{R}^{--}} \equiv \mu_{3}
$$

as follows from the superpotential (2). This relation can be further affected by the contributions of the nonrenormalizable operators in $W_{\mathrm{NR}}$.

The Yukawa interactions of doubly charged Higgsinos read as

$$
\mathcal{L}_{Y}=-2 Y_{L L}^{i j} \bar{L}_{i L}^{c} \tilde{\delta}_{L}^{++} \tilde{L}_{j L}-2 Y_{L L}^{i j} \bar{L}_{i R}^{c} \tilde{\Delta}_{R}^{--} \tilde{L}_{j R}
$$

as follows from the superpotential (2). We consider only the flavor-diagonal Yukawas $(i=j)$, for simplicity. The advantages of studying the production and decays of the doubly charged Higgsinos are evident. Their masses and interactions do not depend on the parameters in other gaugino and Higgsino sectors. In addition, $\tilde{\Delta}_{L}^{--}$and $\tilde{\Delta}_{R}^{--}$ do not mix with each other, so their interactions depend on a small set of unknowns. In fact, the only parameters are their masses $M_{\tilde{\Delta}_{L}^{--}}=M_{\tilde{\Delta}_{R}^{--}}$and leptonic Yukawa couplings $Y_{L L}^{i i}$, which we denote by $Y_{L L}$ from here on. In what follows we forgo further details of the model and proceed directly to analyze the production and decays of the doubly charged Higgsinos at the Tevatron. For additional details about the model, including discussion of the singly charged charginos and neutralinos, as well as the scalar lepton sector, we refer the reader to our previous work [17].

\section{PRODUCTION AND DECAY OF DOUBLY CHARGED HIGGSINOS AT THE TEVATRON}

We focus now on the production modes for doubly charged Higgsinos at the Tevatron. Single as well as pair production of the doubly charged Higgsinos can take place through the $s$-channel exchange of the relevant gauge bosons in the model. The pair-production process at the Tevatron $p \bar{p} \rightarrow \tilde{\Delta}^{++} \tilde{\Delta}^{--}$proceeds with $s$-channel $\gamma$ and $Z_{L, R}$ exchanges, and the single production, $p \bar{p} \rightarrow \tilde{\chi}_{1}^{+} \tilde{\Delta}^{--}$, is dominated by $s$-channel $W_{L, R}$ exchanges. Both processes are initiated by quark-antiquark annihilation at the parton level at the Tevatron.

These doubly and singly charged fermions subsequently decay via a chain of cascades until the lightest neutralino $\tilde{\chi}_{1}^{0}$ is reached. In general, the 2-body decay modes of doubly charged Higgsinos are given by

(i)

$$
\tilde{\Delta}^{--} \rightarrow \tilde{\ell}^{-} \ell^{-},
$$

$$
\tilde{\Delta}^{--} \rightarrow \Delta^{--} \tilde{\chi}_{i}^{0},
$$

$$
\tilde{\Delta}^{--} \rightarrow \tilde{\chi}_{i}^{-} \Delta^{-}
$$

$$
\tilde{\Delta}^{--} \rightarrow \tilde{\chi}_{i}^{-} W^{-},
$$

whose decay products further cascade into lower mass daughter particles of which leptons are of particular interest. Clearly, pair-produced doubly charged Higgsinos can lead to $4 \ell+\mathbb{E}_{T}$ final states, whereas singly produced doubly charged Higgsinos can give rise to $3 \ell+\mathbb{E}_{T}$ signals.

The possibility of light observable doubly charged Higgs bosons has been explored extensively by both phenomenological analyses [20] and experimental investigations, and they resulted in constraining the two-dimensional parameter space spanned by the doubly charged Higgs mass and the $\Delta L=2$ coupling [21]. We hereon concentrate on accessible decay channels devoid of Higgs bosons. Thus we assume that triplet Higgs bosons are heavier and degenerate in mass, which renders them kinematically inaccessible for decay modes of the relatively lighter doubly charged Higgsinos. For the numerical estimates we have considered a representative point in the LRSUSY parameter space, favorable to observing light doubly charged Higgsinos, as tabulated in Table I. We chose a value for $M_{B-L}$ significantly lower than other supersymmetry breaking parameters because it corresponds to the LSP as light, and mostly bino, thus obeying constraints from the relic density analysis for the neutralino [5]. This choice allows for light doubly charged fermions, of masses accessible at the Tevatron. The composition of the lightest neutralinos also affects the 3-body decay (Fig. 1). As we increase $M_{B-L}$, we note that the 3-body decay mode $\left(l l \tilde{\chi}_{2}^{0}\right)$ becomes suppressed even for lower values of $\mu_{3}$ and eventually goes to zero for increasing values of $M_{B-L}$. Seen this way, our choice is a conservative choice and a larger value would strengthen the signal. A quick look at the resulting mass spectrum for the sparticles suggests that the chargino states as well as the scalar leptons are also heavier than the doubly charged Higgsinos, and hence, the favorable decay channel for $\tilde{\Delta}$ (for relatively light Higgsinos) would be the 3-body decays, which would proceed dominantly through off-shell sleptons: $\tilde{\Delta}^{--} \rightarrow$ $\tilde{\ell}^{\star-} \ell^{-} \rightarrow \ell^{-} \ell^{-} \tilde{\chi}_{i}^{0}$, where $\tilde{\chi}_{i}^{0}$ is decided by the allowed kinematic phase space. In addition, the doubly charged 
TABLE I. The numerical values assigned to the model parameters in defining the sample point SPA. In the list, $M_{B-L}, M_{L}$, and $M_{R}$ are, respectively, the masses of $U(1)_{B-L}, S U(2)_{L}$, and $S U(2)_{R}$ gauginos. The VEVs of the left-handed Higgs triplets are taken as $v_{\Delta_{L}} \sim v_{\delta_{L}} \simeq$ $10^{-8} \mathrm{GeV}$. For gauge couplings we take $g_{L}=g_{R}=g$, and for Yukawas we take $Y_{L L}=0.1$. Masses of some light eigenstates are shown in the gable.

\begin{tabular}{cl}
\hline \hline & SPA \\
\hline Fields & $M_{B-L}=25 \mathrm{GeV}, M_{L}=M_{R}=250 \mathrm{GeV}$, \\
& $\tan \beta=5, v_{\Delta_{R}}=3000 \mathrm{GeV}, v_{\delta_{R}}=1000 \mathrm{GeV}$, \\
& $\mu^{(11)}=\mu^{(22)}=1000 \mathrm{GeV}, \mu_{3}=200 \mathrm{GeV}$ \\
$\tilde{\chi}_{i}^{0}(i=1,3)$ & $92.2,120.9,200 \mathrm{GeV}$ \\
$\tilde{\chi}_{i}^{ \pm}(i=1,3)$ & $200,250.9,934.7 \mathrm{GeV}$ \\
$W_{R}, Z_{R}$ & $2090.4,3508.5 \mathrm{GeV}$ \\
$\left(\tilde{e}_{L}, \tilde{e}_{R}\right),\left(\tilde{\mu}_{L}, \tilde{\mu}_{R}\right),\left(\tilde{\tau}_{1}, \tilde{\tau}_{2}\right)$ & $(402,402 \mathrm{GeV}),(402,402 \mathrm{GeV}),(401,406 \mathrm{GeV})$ \\
\hline \hline
\end{tabular}

Higgsinos can have a 3-body decay through the heavy offshell charginos. We have explicitly checked that the 3-body decay of the doubly charged Higgsinos through the heavy off-shell charginos or $W$ bosons is quite suppressed [unless the $\Delta L=2$ coupling $Y_{L L}$ is fine-tuned to a very low value $\left(<10^{-5}\right)$ ] with respect to the 3-body decay through the off-shell sleptons, and can be safely neglected. In Fig. 1 we show the relative branching ratios corresponding to the 3body decays of light doubly charged Higgsinos as a function of their mass. We can see that for a relatively light $\tilde{\Delta}$ of mass $M_{\tilde{\Delta}}<150 \mathrm{GeV}$ the mode $\ell \ell \tilde{\chi}_{2}^{0}$ is the dominant one due to the compositions of light neutralinos, despite the fact that $\tilde{\chi}_{2}^{0}$ is heavier than the LSP. Nevertheless, it falls rapidly when $M_{\tilde{\Delta}}>150 \mathrm{GeV}$, and the mode $\ell \ell \tilde{\chi}_{1}^{0}$ takes

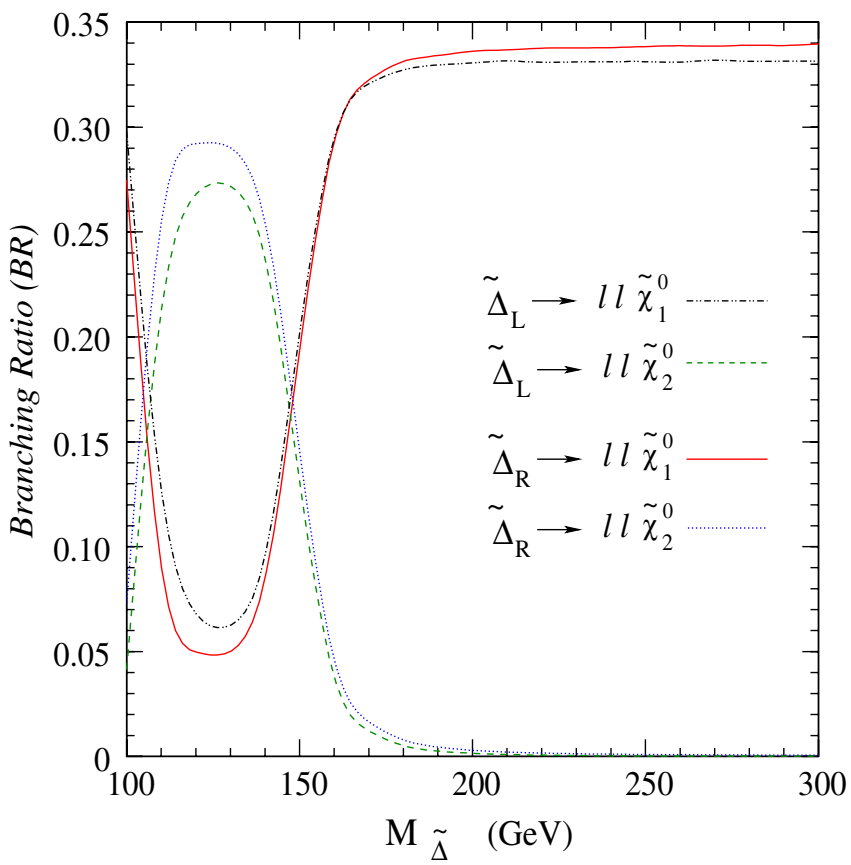

FIG. 1 (color online). The 3-body decay branchings of $\tilde{\Delta}^{--}$ and $\tilde{\Delta}^{++}$for the SPA parameter set, but allowing $\mu_{3}$ to vary. The branching ratios change mainly with what neutralino, $\tilde{\chi}_{1}^{0}$ or $\tilde{\chi}_{2}^{0}$, is produced along with SSSF lepton pairs $(\ell=e$ or $\mu)$. over. Consequently, one writes

$$
\operatorname{BR}\left(\tilde{\Delta}_{L / R}^{--} \rightarrow \ell_{i}^{-} \ell_{i}^{-} \tilde{\chi}_{1}^{0}\right) \simeq \frac{1}{3}, \quad m_{\tilde{l}_{i}}>M_{\tilde{\Delta}^{--}},
$$

where $i=e, \mu$, and $\tau$. One notes that only the 3-body decay channel is allowed when $m_{\tilde{\ell}_{i}}>M_{\tilde{\Delta}}$. We discuss the chargino decays later when analyzing the singleproduction mode.

We now focus separately on the signals at the Tevatron arising from the pair and single productions of these doubly charged Higgsinos.

\section{A. Pair production of doubly charged Higgsinos}

The pair production of doubly charged Higgsinos at the Tevatron occurs through the $s$-channel exchanges of the neutral gauge bosons in the model, viz., the $\gamma, Z$ and the new (heavy) $Z$ boson $\left(Z_{R}\right)$. Since $Z_{R}$ is heavy, it has a negligible contribution to the production at the Tevatron. It is important to note that the production cross section of the doubly charged Higgsino which is a fermion is much larger than that of a doubly charged Higgs of the same mass. The production rate is mainly determined by the fact that the photon couples to the two units of charge carried by the doubly charged Higgsino, and there is a kinematic suppression in the phase space due to the mass of the doubly charged state. Therefore, we can safely assume that a single representative point will be sufficient to present the essential features of the signal one can expect at the Tevatron. In Fig. 2 we plot production cross sections for $\tilde{\Delta}^{--}$for both chiralities and exchanged gauge bosons. It is seen that the cross section is quite sizable for sufficiently light doubly charged Higgsinos: It starts at $\sim 10^{3} \mathrm{fb}$ at $M_{\tilde{\Delta}} \simeq 100 \mathrm{GeV}$ and stays above $\sim 10 \mathrm{fb}$ for $M_{\tilde{\Delta}}$ up to $250 \mathrm{GeV}$. However, it falls rapidly below $\sim 1 \mathrm{fb}$ for masses beyond $M_{\tilde{\Delta}}=300 \mathrm{GeV}$.

The doubly charged Higgsinos decay according to (6) into two same-sign same-flavor (SSSF) leptons and the LSP $\tilde{\chi}_{1}^{0}$. This decay pattern gives rise to final states involving four isolated leptons of the form $\left(\ell_{i}^{-} \ell_{i}^{-}\right)\left(\ell_{j}^{+} \ell_{j}^{+}\right)$: 


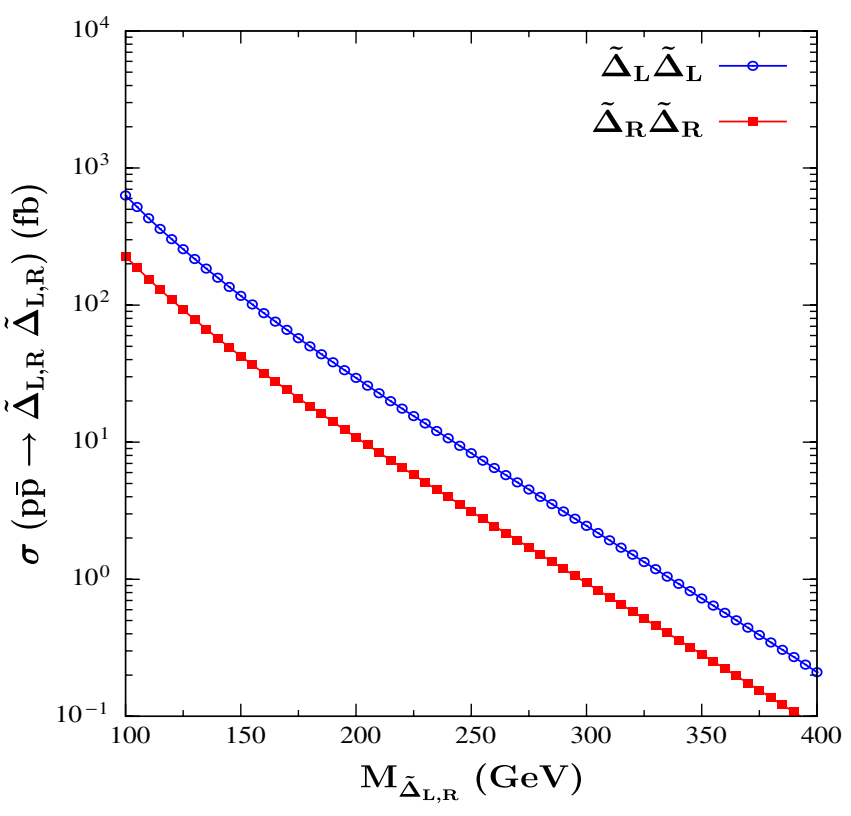

FIG. 2 (color online). The pair-production cross sections for doubly charged Higgsinos of either chirality at the Tevatron. The plots are performed by using the parameter sets SPA except that $M_{\tilde{\Delta}^{--}} \equiv \mu_{3}$ is allowed to vary from 100 up to $400 \mathrm{GeV}$.

$$
p p \rightarrow \tilde{\Delta}^{++} \tilde{\Delta}^{--} \rightarrow\left(\ell_{i}^{+} \ell_{i}^{+}\right)+\left(\ell_{j}^{-} \ell_{j}^{-}\right)+\mathbb{H}_{T},
$$

where $\ell_{i}$ and $\ell_{j}$ are not necessarily identical lepton flavors, and where $\ell_{i}, \ell_{j}=e, \mu$, and $\tau$.

The $4 \ell+\mathbb{E}_{T}$ signal receives contributions from the pair production of both chiral states of the doubly charged Higgsino. Since at the Tevatron it is difficult to determine chiralities of particles, it is necessary to add up their individual contributions to obtain the total number of events. This yields a rather clean and robust $4 \ell+$ missing $E_{T}$ signal with highly suppressed SM background. One finds that the SM cross section with tetraleptons, where $\ell_{i}=e$ and $\ell_{j}=\mu$, in (7) and with large missing transverse energy $\left(\mathscr{E}_{T} \geq 25 \mathrm{GeV}\right)$, receives the dominant contribution from $t \bar{t}$ production, and, to a lesser extent, from the pair production of gauge bosons, $W W$ and $W Z$.

For the numerical analysis, we have included the LRSUSY model into CALCHEP 2.4.5 [22] and generated the event files for the production and decays of the doubly charged Higgsinos using the CALCHEP event generator. The event files are then passed through the CALCHEP + PYTHIA interface, where we include the effects of both initial and final state radiations using PYTHIA [23] switches to smear the final states. We use the leading order CTEQ6L [24] parton distribution functions for the (anti)quarks in (anti) protons. We employ the jet cone algorithm implemented in PYTHIA through the subroutine PYCELL. We assume that the minimum summed the jet transverse energy $E_{T}$ [consisting of all calorimetric cells within the cone of radius $\Delta R=0.7$ in the $(\eta, \phi)$ plane] must be $15 \mathrm{GeV}$ to qualify to be a jet.
The final state leptons are considered to be isolated if they are well resolved from the jets by demanding $\Delta R_{\ell_{i} J} \geq 0.4$.

In addition, for triggering and enhancing the $4 \ell+\not \mathbb{E}_{T}$ signal, we impose the following kinematic cuts [25]:

(i) The charged leptons in the final state must respect the rapidity cut $\left|\eta_{\ell}\right|<2.0$.

(ii) The charged leptons in the final state (arranged according to their $p_{T}$ 's) must satisfy $p_{T}\left(\ell_{1}\right)>$ $11 \mathrm{GeV}, p_{T}\left(\ell_{2}\right)>7 \mathrm{GeV}$. and $p_{T}\left(\ell_{3,4}\right)>5 \mathrm{GeV}$.

(iii) To ensure proper resolution between the final state leptons we demand $\Delta R_{\ell_{i} \ell_{j}}>0.2$ for each pair of leptons, where $\Delta R=\sqrt{(\Delta \phi)^{2}+(\Delta \eta)^{2}}$, with $\phi$ being the azimuthal angle.

(iv) The missing transverse energy must obey $\mathbb{E}_{T}>$ $25 \mathrm{GeV}$.

(v) The pairs of oppositely charged leptons of the same flavor have at least $10 \mathrm{GeV}$ invariant mass.

The SM background yielding tetralepton final states receives the dominant contribution from the $t \bar{t}$ production, which can be significantly suppressed by demanding at least two same-sign muons and two same-sign electrons in the final state. This removes the unwanted large contributions produced at the $Z$ peak. We simulate the SM background using PYTHIA and list the cross sections in Table II. These events are passed through the same kinematic cuts used for the signal. The background is quite small when compared with the signal generated by doubly charged Higgsino pairs. Indeed, as seen from Fig. 2, the SM background nears the signal only when $M_{\tilde{\Delta}} \sim$ $400 \mathrm{GeV}$. This manifest dominance of the signal makes this channel a highly promising one for an efficient and clean disentanglement of LRSUSY effects.

In Fig. 3 we plot the total event cross sections (after applying the kinematic cuts mentioned above). For the four-lepton plus missing energy signal we have specifically chosen the $2 \mu^{-}+2 e^{+}+\mathbb{E}_{T}$ final state. The total cross section for a given final state is obtained by summing over contributions of doubly charged Higgsinos of either chirality. We find that the signal cross section is around $3 \mathrm{fb}$ for $M_{\tilde{\Delta}}=150 \mathrm{GeV}$ and exhibits an increase for slightly heavier $\tilde{\Delta}(\simeq 175 \mathrm{GeV})$ before the kinematic phase space suppression takes over, as the mass of $\tilde{\Delta}$ is further increased. This is quite expected, since the branching frac-

TABLE II. The SM background cross sections for $2 \mu^{-} 2 e^{+}+$ $\mathscr{E}_{T}$ and $2 \mu^{-} e^{+}+\mathscr{E}_{T}$ in the final state at the Tevatron. We have also put a $b$-jet veto (assuming $b$-tagging efficiency to be $50 \%$ ) to suppress the background from the production of $t \bar{t}$.

\begin{tabular}{lll}
\hline \hline & SM background \\
Final states & $2 \mu^{-} 2 e^{+}+\mathscr{E}_{T}+X$ & $2 \mu^{-} 2 e^{+}+\mathscr{E}_{T}+X$ \\
\hline$W W$ and $W Z$ & $0.02 \mathrm{fb}$ & $0.17 \mathrm{fb}$ \\
$t \bar{t}$ & $0.14 \mathrm{fb}$ & $3.58 \mathrm{fb}$ \\
Total & $0.16 \mathrm{fb}$ & $3.75 \mathrm{fb}$ \\
\hline \hline
\end{tabular}




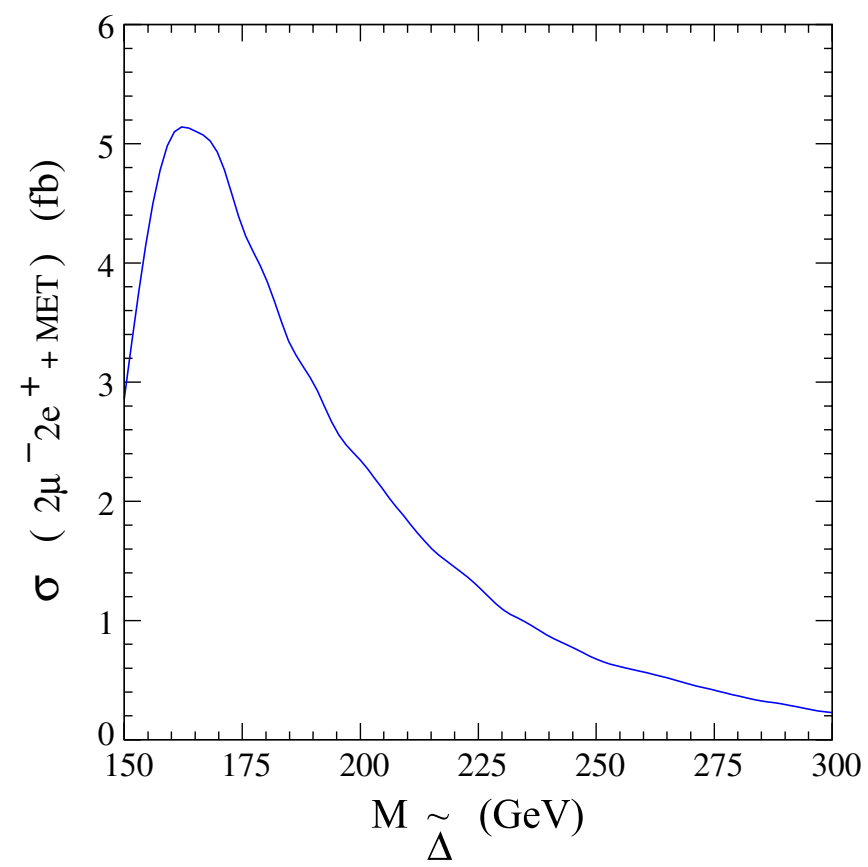

FIG. 3 (color online). The signal cross section for the $2 \mu^{-} 2 e^{+}+\mathbb{E}_{T}$ final state at the Tevatron (after selection cuts) in the LRSUSY model.

tion for the 3-body decay of $\tilde{\Delta}$ to $\ell \ell \tilde{\chi}_{1}^{0}$ depends on the mass of $\tilde{\Delta}$ as shown in Fig. 1. The other decay channel of $\tilde{\Delta}$ decay is $\ell \ell \tilde{\chi}_{2}^{0}$ which also gives two SSSF leptons, with the $\tilde{\chi}_{2}^{0}$ decaying further to $\ell^{+} \ell^{-} \tilde{\chi}_{1}^{0}$ or $q \bar{q} \tilde{\chi}_{1}^{0}$. This channel also contributes to the signal, as the lepton pairs coming from the $\tilde{\chi}_{2}^{0}$ decay are very soft (due to the small mass difference between $\tilde{\chi}_{2}^{0}$ and $\tilde{\chi}_{1}^{0}$ ), and might fail the selection cuts. Even then the contributions are very small compared to the signal arising from $\tilde{\Delta} \rightarrow \ell \ell \tilde{\chi}_{1}^{0}$. The total cross section lies between $2-3 \mathrm{fb}$ for $M_{\tilde{\Delta}}=200 \mathrm{GeV}$ but falls rapidly below $1 \mathrm{fb}$ for $M_{\tilde{\Delta}}>250 \mathrm{GeV}$ for the signal $2 \mu^{-} 2 e^{+}+$ $\mathscr{E}_{T}$. But if we consider no charge identification and just take $2 \mu 2 e+\mathscr{E}_{T}$, then the total cross section is approximately twice the one from above. In principle, one can also work with final states where one of the lepton flavors is $\tau$. Then one needs to fold in the efficiencies for $\tau$ identification at the Tevatron to get the correct event rates.

The Tevatron has already collected data samples with integrated luminosities between $2-3 \mathrm{fb}^{-1}$. With such a robust final state one can expect excellent discovery potential for light doubly charged Higgsinos. Also, the fact that the production rate for such a final state is mainly governed by the mass of $\tilde{\Delta}$ should give us a very strong constraint on the mass of the doubly charged Higgsinos.

In Fig. 4 we choose $M_{\tilde{\Delta}}=200 \mathrm{GeV}$ for the signal analysis and we show some characteristic kinematic features unique to the model when compared with other new physics studies. In Fig. 4(a) we plot the binwise distributions for the invariant masses of the lepton pairs of the signal $2 \mu^{-} 2 e^{+}+\mathscr{E}_{T}$. These plots manifestly show differences between the SSSF and opposite-sign different-flavor (OSDF) lepton pairs with respect to their invariant mass distributions. This is because the SSSF leptons originate from the cascade decay of a single doubly charged Higgsino whereas OSDF lepton configurations are formed
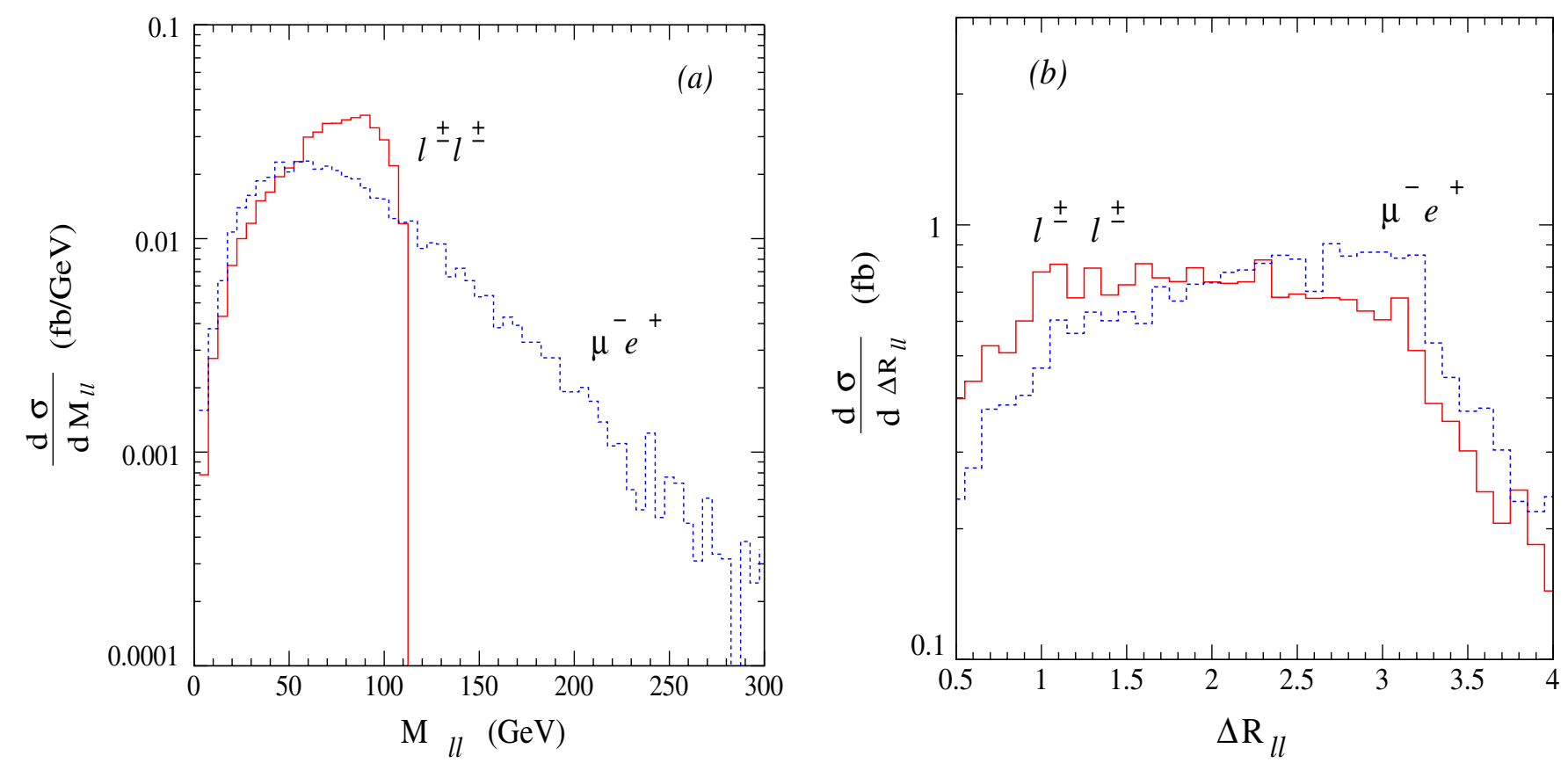

FIG. 4 (color online). The differential cross section of the tetralepton signal with respect to (a) the dilepton invariant mass and (b) $\Delta R$ of a dilepton pair, for $M_{\tilde{\Delta}}=200 \mathrm{GeV}$. The bin sizes in (a) and (b) are $5 \mathrm{GeV}$ and 0.1 , respectively. 
by two isolated leptons, one originating from $\tilde{\Delta}^{--}$, the other from $\tilde{\Delta}^{++}$. For similar reasons, the SSSF lepton pairs exhibit a sharp kinematic edge in their $M_{\ell \ell}$ distributions whereas the OSDF lepton pairs do not. The reason is that SSSF lepton pairs originate from the cascade decay of the same $\tilde{\Delta}$. Since the dilepton invariant mass does not change under boosts, this edge can be well approximated by the formula (in the rest frame of the decaying particle)

$$
M_{\ell^{ \pm} \ell^{ \pm}}^{\max }=\sqrt{M_{\tilde{\Delta}}^{2}+M_{\tilde{\chi}_{1}^{0}}^{2}-2 M_{\tilde{\Delta}} E_{\tilde{\chi}_{1}^{0}},}
$$

where $E_{\tilde{\chi}_{1}^{0}}$ is the energy of the LSP. This formula yields an edge in the invariant mass distribution of the SSSF lepton pairs at the bin around $M_{\ell^{ \pm} \ell^{ \pm}}=M_{\tilde{\Delta}}-M_{\tilde{\chi}_{1}^{0}}$ (in agreement with the numerical values corresponding to the SPA point given in Table I) in the case of the 3-body decay of $\tilde{\Delta}$, as can be seen in Fig. 4(a). This corresponds to the situation when the LSP is produced at rest in the frame of $\tilde{\Delta}$. The effect of the changing of the value of $M_{B-L}$ in this analysis is that the lightest neutralino mass would change [18], so the allowed phase space would change very minutely and the position of the kinematic edge in Fig. 4(a) would change. In addition the 3-body partial decay width for the doubly charged Higgsino would change, but we are mainly concerned with the branching ratio, which is about $100 \%$, and this is not affected.

A comparison of the SSSF and OSDF leptonic spectra qualifies to be a viable probe of the doubly charged Higgsinos. First, the edge in the SSSF dilepton invariant mass distribution yields a clear hint of a $\Delta L=2$ interaction and a doubly charged field in the underlying model of "new physics." The distributions of the OSDF dileptons exhibit no such edge for the reasons mentioned before. Second, Fig. 4(b) manifestly shows the differences in the binwise distribution of the spatial resolution $\Delta R$ between the charged lepton pairs for the cases indicated on the curves (with $l^{ \pm} l^{ \pm}$standing for $\mu^{-} \mu^{-}$or $e^{+} e^{+}$). The SSSF leptons have distributions peaked at low values of $\Delta R$ (as they originate from one single doubly charged Higgsino $\tilde{\Delta}$ ) whereas the OSDF leptons exhibit pronounced distributions at higher values of $\Delta R$ (as they originate from different Higgsinos, one from $\tilde{\Delta}^{--}$and the other from $\tilde{\Delta}^{++}$). To this end, SSSF leptons with small spatial separation qualify to be a direct indication of the doubly charged Higgsinos in the spectrum. In addition to the kinematic edge highlighted for the dilepton invariant mass, this feature is a clear-cut signal of extended SUSY models as it is absent in the MSSM or in any of its extensions that contain only singly charged fields.

\section{B. Associated productions of doubly charged Higgsinos and charginos}

In this section we study productions and decays of doubly charged Higgsinos in association with the lightest chargino. The process under consideration has the form

$$
p \bar{p} \rightarrow \tilde{\Delta}^{--} \tilde{\chi}_{1}^{+} \rightarrow\left(\ell_{i}^{-} \ell_{i}^{-}\right)+\ell_{j}^{+}+\mathbb{E}_{T},
$$

where $\ell_{i}$ is not necessarily identical to $\ell_{j}$. This scattering process proceeds with the $s$-channel $W_{L, R}$ exchange, and yields invariably a trilepton signal, which has long been considered as a strong indication of SUSY, in general [26]. In Fig. 5 we plot the cross section for the associated production of the $\tilde{\Delta}^{ \pm \pm}$which should give us a $3 \ell+\mathbb{E}_{T}$ signal. The cross section for singly produced doubly charged Higgsino turns out to be small compared to the pair-production cross section. The production is completely dominated by the left-chiral doubly charged state for the model parameters (SPA) shown in Table I. Indeed, the right-chiral state is produced via the $s$-channel exchange of the heavy $W_{R}$ boson, and it is thus strongly suppressed. There is also additional suppression due to the composition of the lightest chargino, which affects the coupling of $W_{R}^{\mu}-\tilde{\chi}_{1}^{+}-\tilde{\Delta}_{R}^{--}$. The couplings of the chargino states to weak gauge bosons and the doubly charged Higgsino are

(i)

$$
W_{L}^{\mu} \tilde{\chi}_{k}^{+} \tilde{\Delta}_{L}^{--}: i g_{L} \gamma^{\mu}\left(V_{k 5}^{\star} P_{L}+U_{k 5} P_{R}\right),
$$

$$
W_{R}^{\mu} \tilde{\chi}_{k}^{+} \tilde{\Delta}_{R}^{--}: i g_{R} \gamma^{\mu}\left(V_{k 6}^{\star} P_{L}+U_{k 6} P_{R}\right),
$$

where $U$ and $V$ are the matrices which diagonalize the

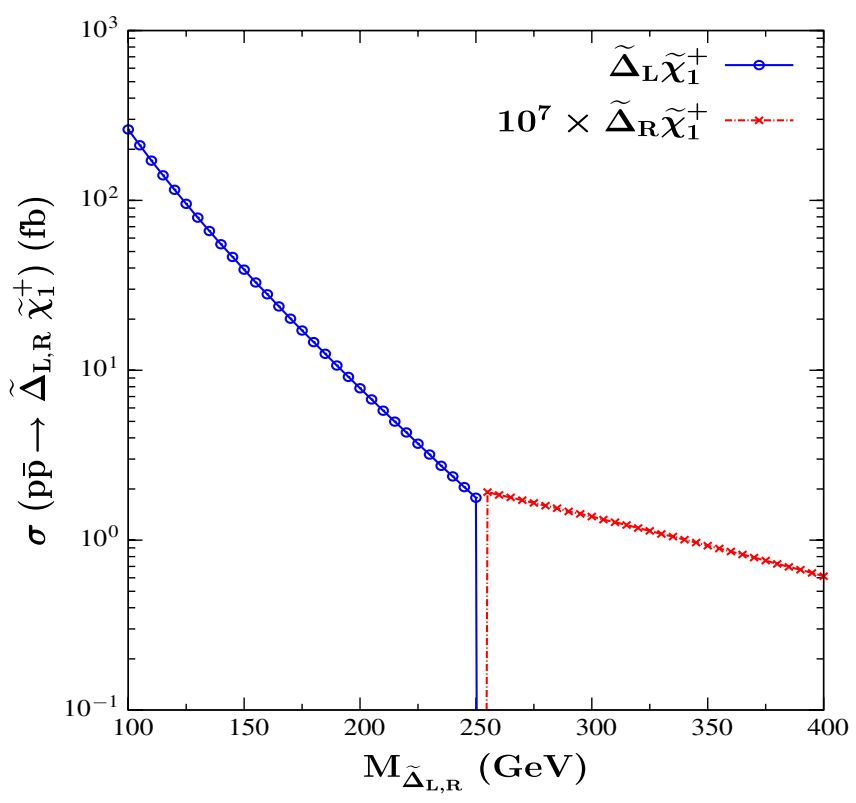

FIG. 5 (color online). The cross sections for productions of $\tilde{\Delta}_{L, R}$ in association with $\tilde{\chi}_{1}^{ \pm}$at the Tevatron. The model parameters are as in SPA in Table I, except that $M_{\tilde{\Delta}^{--}} \equiv \mu_{3}$ is varied from 100 up to $400 \mathrm{GeV}$. 
chargino mass matrix. The production cross section becomes negligible around $M_{\tilde{\Delta}}=250 \mathrm{GeV}$, at which the composition of the lightest chargino changes abruptly. More precisely, the lightest chargino is dominantly triplet Higgsino for $\mu_{3} \leq 250 \mathrm{GeV}$, for which the coupling $W-$ $\tilde{\chi}_{1}^{+}-\tilde{\Delta}_{L}$ is maximal. For $\mu_{3}>M_{L}$, there exists a nonnegligible gaugino contamination in the lightest chargino, and the $W-\tilde{\chi}_{1}^{+}-\tilde{\Delta}_{L}$ depends on the left-triplet VEV, which suppresses it below any measurable level. This also explains the fact that the associated single production of $\tilde{\Delta}$ with the lightest chargino will identically vanish as soon as its gaugino component starts becoming non-negligible. Thus, the $3 \ell+\mathbb{E}_{T}$ signal with a pair of SSSF leptons depends strongly on the composition of the chargino.

Consequently, it suffices to use only the left-chirality doubly charged Higgsino production in association with the lightest chargino $\tilde{\chi}_{1}^{+}$. Nevertheless, we note that for the choice of parameters in (SPA), as the composition of the chargino changes around $\mu_{3} \geq 250 \mathrm{GeV}$, the associated production of $\tilde{\chi}_{2}^{+} \tilde{\Delta}_{L}^{--}$begins to dominate. Figure 5 shows that, for the entire SPA parameter space with varying $\mu_{3}$, the left-chirality doubly charged Higgsino produced in association with the lightest chargino yields a reasonable cross section for small Higgsino masses and yields approximately $2 \mathrm{fb}$ cross section for $\tilde{\Delta}^{ \pm \pm}$as heavy as $M_{\tilde{\Delta}} \sim$ $250 \mathrm{GeV}$. For comparison, we also include the cross section for the right-handed Higgsino, which is small, even when $\mu_{3}>250 \mathrm{GeV}$ (because of the strong $s$-channel suppression and nature of the composition of the lightest chargino, as pointed out earlier). One notes here that, since the chargino couplings to $\tilde{\Delta}_{L / R}$ depend on the entries in the mixing matrices of charginos, the input parameters in Table I play a crucial role in determining the production cross section. The cross section for $p \bar{p} \rightarrow \tilde{\Delta}_{L}^{--} \tilde{\chi}_{1}^{+}$is around $8 \mathrm{fb}$ for the SPA parameter set. As in pairproduction mode, the $\tilde{\Delta}^{--}$decays again into a pair of SSSF leptons and the LSP following (6). However, the chargino, too, exhibits a 3-body decay through off-shell sleptons with almost $100 \%$ branching into the 3-body final state of neutrino, lepton, and the LSP for the SPA parameter set with $M_{\tilde{\Delta}} \geq 175 \mathrm{GeV}$. For lower values of $\mu_{3}=$ $M_{\tilde{\Delta}}$, however, the mode $\bar{\nu}_{\ell} \ell^{+} \tilde{\chi}_{2}^{0}$ competes. The above decays yield a $3 \ell+\mathscr{E}_{T}$ final state, where the missing transverse energy arises from the undetected LSP and the neutrino. For the benchmark point SPA, the signal acquires all the contribution from the left-chirality state production.

The single $\tilde{\Delta}^{--}$production gives rise to a trilepton signal at the Tevatron experiments. In the numerical analysis, following the same notation and same kinematic cuts as in the previous section (for the three leading charged leptons), we illustrate the case where $\ell_{i}=\mu$ and $\ell_{j}=e$. Thus, we know that the $e^{+}$always comes from the chargino while the same-sign muons originate from the doubly charged Higgsino. In parallel to the analysis of the $4 \ell+$
$\mathscr{E}_{T}$ signal in the previous section, the characteristic kinematic features remain very similar to the ones that have already been covered by discussions in the previous section. In particular, the distributions of the SSSF leptons are quite similar to the ones for the $4 \ell+\not \mathbb{E}_{T}$ signal. This is actually expected since SSSF leptons are exclusively generated by the decays of the doubly charged Higgsino, a common feature for both tetralepton and trilepton final states.

However, we note that the SM background is substantial for the trilepton final state at the Tevatron energies. It stands at the $3.75 \mathrm{fb}$ level with the most dominant source being the $t \bar{t}$ production. We list the major backgrounds in Table II. We have plotted the signal + SM cross section in Fig. 6 by considering specifically the $2 \ell_{i}^{-}+\ell_{j}^{+}+\mathscr{E}_{T}$ signal with $\ell_{i}=\mu$ and $\ell_{j}=e$. It shows that an integrated luminosity of $10 \mathrm{fb}^{-1}$ does not give a $3 \sigma$ signal over the background in the trilepton channel for SPA. For an integrated luminosity of $16 \mathrm{fb}^{-1}$ the signal reach at $2 \sigma$ is for $M_{\tilde{\Delta}} \lesssim 178 \mathrm{GeV}$ while the reach is only extended at most to $M_{\tilde{\Delta}} \lesssim 154-162 \mathrm{GeV}$ for a $3 \sigma$ signal. The SM background can be reduced further to $1.52 \mathrm{fb}$ by choosing a set of larger cuts for $p_{T}$, namely $\{20,15,10\} \mathrm{GeV}$ (taken in the same order as before). This helps in extending the reach for a $2 \sigma$ signal to doubly charged Higgsino masses of $M_{\tilde{\Delta}} \lesssim$ $190 \mathrm{GeV}$ while the $3 \sigma$ reach stands at $M_{\tilde{\Delta}} \lesssim 173 \mathrm{GeV}$.

Nonetheless, the pair production of doubly charged Higgsinos for the same representative point SPA gives at least 2 (8) events for the tetralepton signal for $M_{\tilde{\Delta}}=$ $280 \mathrm{GeV}$, for an integrated luminosity of 4(16) $\mathrm{fb}^{-1}$ where

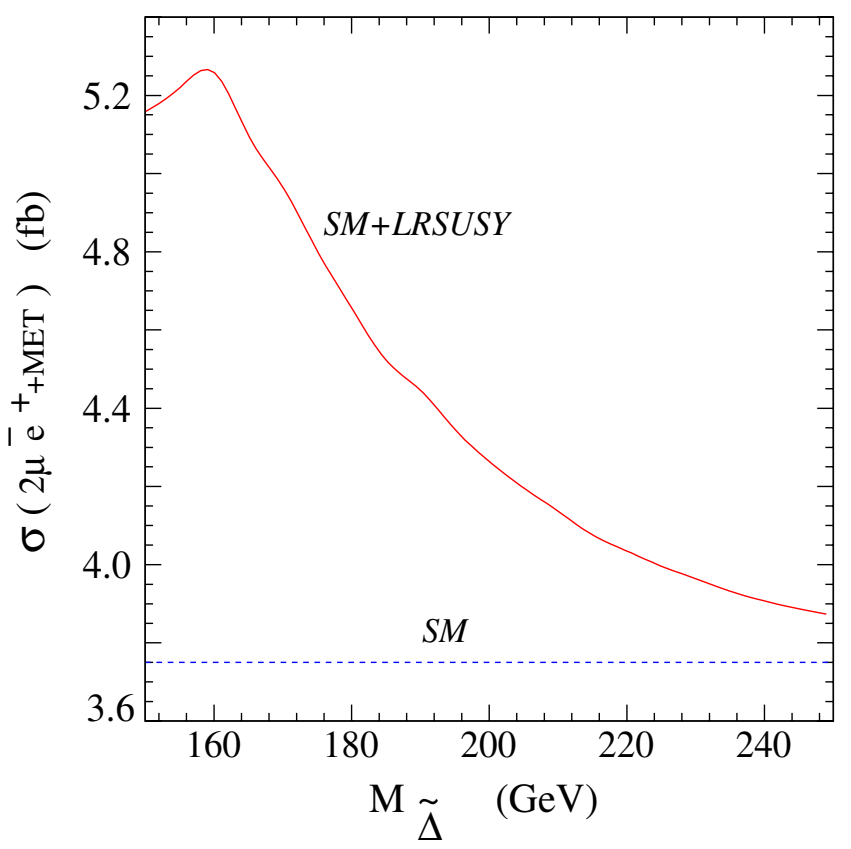

FIG. 6 (color online). The cross section for the $2 \mu^{-} e^{+}+\mathbb{E}_{T}$ final state at the Tevatron (after selection cuts). 
0 (3) SM events are expected, giving thus a clear discovery signal for the doubly charged exotica.

\section{DISCUSSIONS AND CONCLUSIONS}

We have studied the Tevatron signals of doubly charged Higgsinos present in the left-right symmetric SUSY models. The doubly charged Higgsinos in the spectrum are a characteristic feature of LRSUSY, which can directly and unambiguously distinguish the model from the MSSM (and its various extensions like NMSSM and the $U(1)^{\prime}$ models) via generic leptonic events observed in collider experiments. We have given a detailed account of the leptonic signals originating from production and decay of (i) doubly charged Higgsino pairs (in Sec. III A) and of (ii) single doubly charged Higgsino plus chargino (in Sec. III B). For the production mode (i), the leptonic final state invariably involves $\left(\ell_{i}^{-} \ell_{i}^{-}\right)+\left(\ell_{j}^{+} \ell_{j}^{+}\right)+\mathbb{E}_{T}$, that is, a pair of SSSF dileptons plus missing energy acquired by the LSP, $\tilde{\chi}_{1}^{0}$. On the other hand, for the production mode (ii) the leptonic final state is composed of $\left(\ell_{i}^{-} \ell_{i}^{-}\right)+\ell_{j}^{+}+\mathscr{E}_{T}$, that is, a trilepton signal.

The simulation study of the pair-production process, characterized by tetralepton final states with SSSF or OSDF structures, provides a unique opportunity to track down the presence of doubly charged fermions in the model of new physics at the $\mathrm{TeV}$ domain. Especially noticeable are the distributions of the SSSF leptons, which can firmly establish if there exists a doubly charged fermion that decays into SSSF leptons plus missing energy (by using the dilepton invariant mass and their spatial proximity). Also important is the fact that the SSSF and OSDF lepton signals well dominate over the SM signal up to relatively large doubly charged Higgsino masses.

The simulation study of the production of single doubly charged Higgsino in association with a chargino, which yields the celebrated trilepton signal, also proves to be an important signature of doubly charged fermions of new physics. Nevertheless, the signal dominates over the SM background only for low Higgsino masses, and one cannot extract the information about doubly charged fermions as confidently as in the tetralepton signal above.

The search program in this work can be extended to a multitude of new physics models at both the qualitative and quantitative levels. Generically, however, the LRSUSY, whose spectrum consists of doubly charged Higgs fermions, stands fundamentally different than the rest as it possesses SSSF proximate dileptons at the final state. In this sense, the analysis in this work shows that, under minimal assumptions about the model and its parameter space SPA, as far as detector acceptance permits, the fingerprints of light doubly charged Higgsinos can be searched in the $4 \mathrm{fb}^{-1}$ of data collected by the CDF and D0 experiments. This requires performing the requisite "event mining" in Tevatron data exclusively for tetralepton final states with SSSF structure. Such exotic structures, if discovered, would provide a spectacular signal for physics beyond SM and MSSM.

\section{ACKNOWLEDGMENTS}

The work of D.D. was supported by the Turkish Academy of Sciences via a GEBIP grant, by the Turkish Atomic Energy Agency via a CERN-CMS grant, and by IZTECH via a 2009 BAP grant. The work of M. F. and I. T. was supported in part by NSERC of Canada under the Grant No. SAP01105354. D. K. G. acknowledges the partial support from the Department of Science and Technology, India under Grant No. SR/S2/HEP-12/2006 . K. H. and S. K. R. gratefully acknowledge support from the Academy of Finland (Project No. 115032). We thank D. Choudhury and B. Mukhopadhyaya for discussions. D. K. G. would like to acknowledge the hospitality provided by the Helsinki Institute of Physics. D. K. G., K. H., and S. K. R. thank the Nordita program TeV scale physics and dark matter for an inspiring atmosphere.
[1] S. Gruenendahl, arXiv:0812.4996v1.

[2] M. Frank, I. Turan, and M. Sher, Phys. Rev. D 71, 113002 (2005); 71, 113001 (2005).

[3] B. Dutta, Y. Mimura, and R. N. Mohapatra, Phys. Rev. D 72, 075009 (2005).

[4] R. N. Mohapatra, Phys. Rev. D 34, 3457 (1986); A. Font, L. Ibanez, and F. Quevedo, Phys. Lett. B 228, 79 (1989); S. Martin, Phys. Rev. D 46, R2769 (1992).

[5] D. A. Demir, M. Frank, and I. Turan, Phys. Rev. D 73, 115001 (2006).

[6] L. Pape and D. Treille, Rep. Prog. Phys. 69, 2843 (2006); M. Spiropulu, arXiv:0801.0318.

[7] R. N. Mohapatra and A. Rašin, Phys. Rev. Lett. 76, 3490
(1996); Phys. Rev. D 54, 5835 (1996); R. Kuchimanchi, Phys. Rev. Lett. 76, 3486 (1996).

[8] P. Minkowski, Phys. Lett. 67B, 421 (1977); R. N. Mohapatra and G. Senjanovic, Phys. Rev. Lett. 44, 912 (1980); Phys. Rev. D 23, 165 (1981); M. Gell-Mann, P. Ramond, and R. Slansky, Supergravity, edited by P. van Nieuwenhuizen et al. (North-Holland, Amsterdam, 1980), p. 315; T. Yanagida, in Proceedings of the Workshop on the Unified Theory and the Baryon Number in the Universe, edited by O. Sawada and A. Sugamoto (KEK, Tsukuba, Japan, 1979), p. 95; S.L. Glashow, in Proceedings of the Summer Institute on Quarks and Leptons, edited by M. Levy et al. (Plenum Press, New 
York, 1980), p. 687.

[9] M. Cvetic and J. Pati, Phys. Lett. 135B, 57 (1984); R. N. Mohapatra, A. Rašin, and G. Senjanović, Phys. Rev. Lett. 79, 4744 (1997); C.S. Aulakh, K. Benakli, and G. Senjanovic, Phys. Rev. Lett. 79, 2188 (1997); C.S. Aulakh, A. Melfo, and G. Senjanovic, Phys. Rev. D 57, 4174 (1998).

[10] R. M. Francis, M. Frank, and C. S. Kalman, Phys. Rev. D 43, 2369 (1991); K. Huitu, J. Maalampi, and M. Raidal, Phys. Lett. B 328, 60 (1994); K. Huitu and J. Maalampi, Phys. Lett. B 344, 217 (1995).

[11] K. Huitu, J. Maalampi, and M. Raidal, Nucl. Phys. B420, 449 (1994).

[12] Z. Chacko and R. N. Mohapatra, Phys. Rev. D 58, 015003 (1998); B. Dutta and R. N. Mohapatra, Phys. Rev. D 59, 015018 (1998).

[13] G. F. Giudice and A. Masiero, Phys. Lett. B 206, 480 (1988).

[14] J. E. Kim and H. P. Nilles, Phys. Lett. 138B, 150 (1984); D. Suematsu and Y. Yamagishi, Int. J. Mod. Phys. A 10, 4521 (1995); M. Cvetic and P. Langacker, Mod. Phys. Lett. A 11, 1247 (1996); V. Jain and R. Shrock, arXiv:hepph/9507238; Y. Nir, Phys. Lett. B 354, 107 (1995); D. A. Demir, Phys. Rev. D 59, 015002 (1998).

[15] K. Huitu, J. Maalampi, A. Pietila, and M. Raidal, Nucl. Phys. B487, 27 (1997); G. Barenboim, K. Huitu, J. Maalampi, and M. Raidal, Phys. Lett. B 394, 132 (1997).

[16] K. Huitu, J. Maalampi, and M. Raidal, Report No. HUSEFT-I-1995-1, 1995.

[17] D. A. Demir, M. Frank, K. Huitu, S. K. Rai, and I. Turan, Phys. Rev. D 78, 035013 (2008).

[18] M. Frank, K. Huitu, and S. K. Rai, Phys. Rev. D 77, 015006 (2008).

[19] B. Dutta, R. N. Mohapatra, and D. J. Muller, Phys. Rev. D 60, 095005 (1999); M. Frank, Phys. Rev. D 62, 053004 (2000); M. Raidal and P. M. Zerwas, Eur. Phys. J. C 8, 479 (1999).

[20] H. Georgi and M. Machacek, Nucl. Phys. B262, 463 (1985); K. Huitu, J. Maalampi, A. Pietila, and M. Raidal, Nucl. Phys. B487, 27 (1997); K. Huitu, P. N. Pandita, and K. Puolamaki, Phys. Lett. B 415, 156
(1997); 423, 97 (1998); S. Chakrabarti, D. Choudhury, R. M. Godbole, and B. Mukhopadhyaya, Phys. Lett. B 434, 347 (1998); K. Huitu, P. N. Pandita, and K. Puolamaki, arXiv:hep-ph/9904388; S. Godfrey, P. Kalyniak, and N. Romanenko, Phys. Rev. D 65, 033009 (2002); J. Maalampi and N. Romanenko, Phys. Lett. B 532, 202 (2002); S. Godfrey, P. Kalyniak, and N. Romanenko, Phys. Lett. B 545, 361 (2002); A. G. Akeroyd and M. Aoki, Phys. Rev. D 72, 035011 (2005); B. Mukhopadhyaya and S. K. Rai, Phys. Lett. B 633, 519 (2006); J. E. Cieza Montalvo, N. V. Cortez, J. Sa Borges, and M. D. Tonasse, Nucl. Phys. B756, 1 (2006); B796, 422 (E) (2008); T. Rommerskirchen and T. Hebbeker, J. Phys. G 34, N47 (2007).

[21] J. Abdallah et al. (DELPHI Collaboration), Phys. Lett. B 552, 127 (2003); G. Abbiendi et al. (OPAL Collaboration), Phys. Lett. B 577, 93 (2003); P. Achard et al. (L3 Collaboration), Phys. Lett. B 576, 18 (2003); D.E. Acosta et al. (CDF Collaboration), Phys. Rev. Lett. 93, 221802 (2004); 95, 071801 (2005); T. Aaltonen (CDF Collaboration), Report No. FERMILAB-PUB-07-709-E.

[22] A. Pukhov, arXiv:hep-ph/0412191.

[23] T. Sjostrand, S. Mrenna, and P. Skands, J. High Energy Phys. 05 (2006) 026.

[24] J. Pumplin, D. R. Stump, J. Huston, H. L. Lai, P. Nadolsky, and W. K. Tung, J. High Energy Phys. 07 (2002) 012; D. Stump, J. Huston, J. Pumplin, W. K. Tung, H. L. Lai, S. Kuhlmann, and J.F. Owens, J. High Energy Phys. 10 (2003) 046; T. Sjostrand, S. Mrenna, and P. Skands, J. High Energy Phys. 05 (2006) 026.

[25] S. Abel et al. (SUGRA Working Group Collaboration), arXiv:hep-ph/0003154.

[26] H. Baer, K. Hagiwara, and X. Tata, Phys. Rev. D 35, 1598 (1987); P. Nath and R. Arnowitt, Mod. Phys. Lett. A 2, 331 (1987); H. Baer and X. Tata, Phys. Rev. D 47, 2739 (1993); H. Baer, C. h. Chen, F. Paige, and X. Tata, Phys. Rev. D 50, 4508 (1994); B. Abbott et al. (D0 Collaboration), Phys. Rev. Lett. 80, 1591 (1998); F. Abe et al. (CDF Collaboration), Phys. Rev. Lett. 80, 5275 (1998); V. D. Barger, C. Kao, and T.j. Li, Phys. Lett. B 433, 328 (1998). 